\title{
Classification of chest radiographs for epidemiological purposes by people not experienced in the radiology of pneumoconiosis
}

\author{
LIZ COPLAND, J BURNS, * AND M JACOBSEN \\ From the Institute of Occupational Medicine, Edinburgh, UK
}

ABSTRACT Under controlled conditions 16 people (eight non-medical) inexperienced in the radiology of occupational lung diseases repeatedly classified 300 selected chest radiographs using the 1971 ILO U/C International Classification of Radiographs of Pneumoconioses. Eight experienced medical readers had previously classified 220 of the selected radiographs for profusion of small rounded opacities. Variability among readers was greater in the experimental panels than among the experienced readers. But the average consistency between pairs of novice readers in their use of the 12 categories of profusion for the same radiographs was similar (about $29 \%$ ) to the average consistency among the experienced readers. Subsequent work with nine of the participants showed that eight of them were able to produce classifications of coal miners' chest radiographs that correlated well with estimates of the miners' exposures to respirable coal mine dust. It is concluded that the ILO classification scheme provides a sound descriptive system for recording the appearances of chest radiographs. Under controlled conditions the scheme may be used for epidemiological studies by those with no specialist knowledge or clinical experience. This presupposes that the radiographs concerned will have been examined previously for diagnostic purposes by a suitably qualified physician.

The film-reading effort required to classify chest radiographs for sound epidemiological studies is time-consuming. Nevertheless, people with no medical qualifications can be trained to produce such classifications $^{1}$; two non-medical readers produced results that agreed more closely with those of their medically qualified trainer than did classifications of the same radiographs by two other experienced radiologists. But all the classifications concerned were supposed to have conformed to a standard system now incorporated in the International Labour Office's Classification of Radiographs of Pneumoconioses. ${ }^{2}$ The question therefore arises: whose interpretation of the classification scheme corresponded more closely to reality-the nonmedical readers' attempts to mimic their medical mentor's views, or the classifications by one or other of the two experts who disagreed among themselves

\footnotetext{
*Present address: National Coal Board, Medical Service, Hobart House, London SW1X 7AE.

Received 9 July 1980

Accepted 13 October 1980
}

and with the trainer? In this context "reality" means abnormality attributable to the inhalation of dust.

The present study was designed to establish whether radiological novices, either with or without medical qualifications, can teach themselves to classify chest radiographs for epidemiological purposes without expert guidance, using only internationally agreed standard films and the accompanying descriptive document produced by the ILO. The plausibility of results has been assessed in terms of variability between readers, comparisons with experienced readers, and correlations with estimates of coal miners' exposures to respirable dust.

\section{Methods}

RECRUITMENT AND INDUCTION OF PANELS Eight people with no medical degrees (the "lay panel"-four nurses, three radiographers, and one retired science graduate) and eight doctors with no special experience in chest radiology were chosen randomly from responses to newspaper advertisements. They were invited to train themselves, under 
controlled conditions, to use the ILO U/C International Classification of Radiographs of Pneumoconioses. ${ }^{2}$ The only teaching aids used in the initial phase of the work were standard radiographs issued by the ILO in 1971 and the text describing the classification scheme. This material was introduced to the participants at inaugural meetings where the aims of the experiment were explained. The lay panel were also given a short demonstration of the radiographic anatomy of the chest (using the standard category 0 radiograph for illustrative purposes) and were provided with a glossary of medical terms used in the ILO booklet. No further help was given, and there were no meetings or discussions between readers during the self-training experiment.

\section{SELF-TRAINING PROTOCOL AND}

CONDITIONS

After the inaugural meetings the 16 readers were presented with 300 selected full-size posteroanterior chest radiographs arranged in random order and in conveniently sized batches. They were asked to classify them by comparing their appearances with ILO standard radiographs and referring only to the ILO descriptive text for guidance. The readers worked alone, without clerical help, for not longer than one three-hour (later two-and-a-half-hour) session in any one week. Two identical blacked-out rooms were used. Background lighting was similar and kept constant. Four-panel viewing boxes were provided with the two panels at the extremities of the box obscured. The luminance of the two central panels was monitored regularly, and light tubes were replaced after about $15 \%$ reductions in luminance had occurred.

Readers were not required to classify any particular number of radiographs per session but were allowed to spend as much time as they thought necessary on each radiograph. An initial comparison of each test radiograph with the standard for category 0 was mandatory. Subsequent classification was to be based primarily on comparisons with other standard radiographs. The same 300 radiographs were presented to them repeatedly in different order and without any indication that they had classified them previously. Time intervals between repeated presentations of the test radiographs varied, and sometimes several batches of other films were classified before a reader was confronted again with the $\mathbf{3 0 0}$ test films. Written comments were solicited on any aspect at every stage of the experiment.

\section{TEST RADIOGRAPHS}

The 300 test radiographs included 60 of men with no occupational exposure to dust and from a wide agerange (17-67 years). The remainder were radiographs of coal miners. They were selected on the basis of classifications made some years earlier ${ }^{3} 4$ by experienced readers, who at that time had been guided by standard radiographs issued by the ILO in 1959. These $\mathbf{2 4 0}$ radiographs comprised:

(a) One hundred and forty films representing a nominally uniform distribution over categories $\mathbf{0}$, $1,2,3, \mathrm{~A}, \mathrm{~B}$, and $\mathrm{C}$;

(b) Fifty films about which eight experienced readers had been divided fairly evenly in their opinions as to whether they were classifiable as category 0 or category 1 ; and

(c) Fifty films about which there had been similar disagreement as to whether they should be classified as category 1 or category 2 .

Of the films in (a) 120 were selected on the basis of relatively low variability among eight experienced readers who had classified them previously ${ }^{3}$ using the 12-point scale for profusion of small opacities; these films were also judged as showing a range of technical quality. The other 20 radiographs in group (a) represented earlier agreements by only two experienced readers. ${ }^{4}$ Nineteen of them had been classified as showing large shadows. The disagreements between experienced readers concerning classifications of films in groups $(b)$ and $(c)$ were often in choice of subcategory adjacent to the main category boundary concerned.

\section{RADIOGRAPHIC CLASSIFICATIONS IN RELATION TO DUST EXPOSURE}

Six of the lay panel and three of the doctors (the "residual panel") continued to classify radiographs under the same conditions after completing the experimental self-training phase. At this stage they were given information about their earlier performances in the form of statistical summaries of results, but the identities of individuals were not disclosed. The difficulties of distinguishing between normal lung markings and early signs of pneumoconiotic lesions were also discussed with them, collectively. The residual panel continued to work under the same protocol. Radiographs presented to them subsequently included 792 that had been classified some months earlier by each of six experienced readers who had based their classifications on standards issued by the ILO in 1968. The films were of 792 miners who had all attended at the fourth of the series of radiological surveys of the National Coal Board's pneumoconiosis field research. Each of these men had attended also at the first surveys, some 15 years earlier, and individuals' exposures to respirable coal mine dust during the roughly 15 -year intervals were known from measurements by the research staff at the collieries. Careful estimates had also been made of exposures to dust before the 
Table 1 Pooled percentage distributions of classifications of test radiographs for small rounded opacities from the third readings by the experimental panels and earlier classifications by eight experienced readers

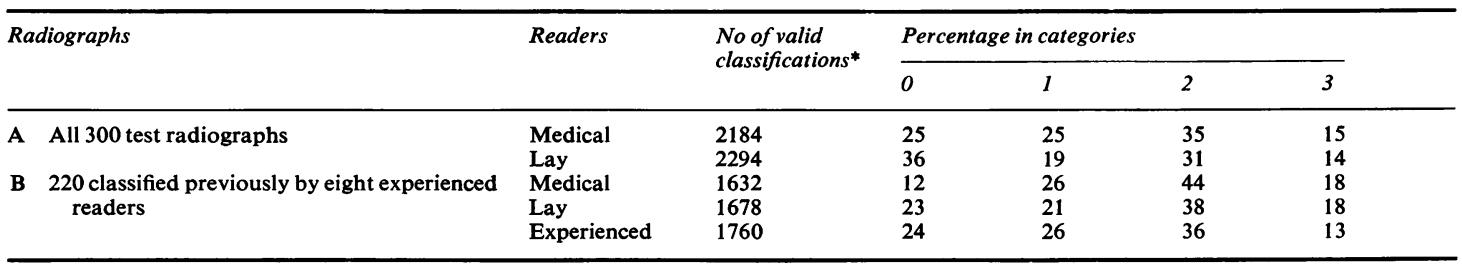

* Reading sheets not completed precisely according to protocol were regarded as invalid and were disregarded in analyses of results.

initial surveys. ${ }^{5}$ Thus there was available for each of the men considered an estimate of his cumulative exposures to respirable coal mine dust, from entry to the industry up to the time when the (fourth) radiological surveys took place.

\section{Results}

Most of the readers' comments related to difficulties in reconciling the written description of classifications in the ILO text with the appearances of the standard radiographs provided. Ambiguities and inconsistencies in the text itself were noted, and there were many complaints alleging inadequacies in some of the standard films. Reading rates were extremely cautious during the first few sessions, varied considerably among readers, but increased steadily as confidence increased, averaging about 60 films per three hours after 16 sessions-that is, 48 hours' reading experience.*

As a group, the medically qualified readers ("medical panel") consistently found a higher prevalence of small rounded opacities than the lay

* Five readers from these experimental panels are continuing regular part-time classification of films for epidemiological purposes. They are now reading 300 films per two-and-a-halfhour session, with clerical help for recording results.

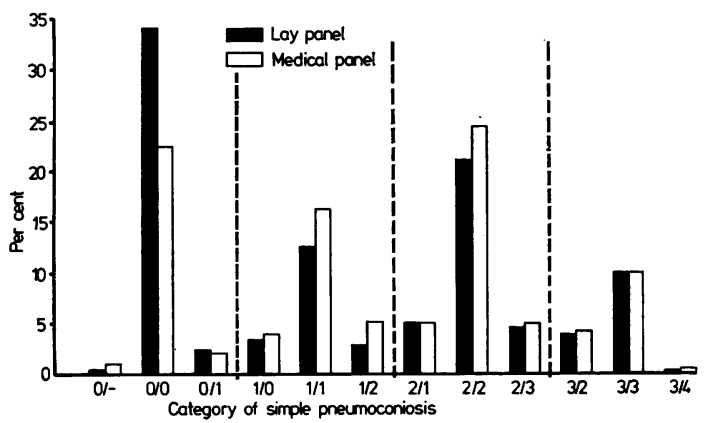

Fig 1 Lay and medical readers' pooled distributions of 300 test radiographs to pneumoconiosis categories for small rounded opacities; third readings. panel, but the average reading patterns tended to converge with experience. Table 1 summarises results from the the third readings in terms of the panels' pooled distributions of classifications to the four main ILO categories. Part A of the table refers to all 300 test radiographs. The corresponding distributions to the categories of the 12-point ILO classification are compared in fig 1 . This shows that the medical panel's fewer classifications into category 0 ( $25 \%$ as compared with $36 \%$ for the lay panel) are not to be explained simply by disagreements on the use of categories $0 / 1$ and $1 / 0$; the medically qualified readers classified more films as showing small rounded opacities in higher categories of profusion.

Part B of table 1 refers to the subset of radiographs for which comparisons are possible with earlier classifications by eight experienced readers. These results indicate that on average the lay readers agreed more closely with the experienced readers than with the self-trained medically qualified readers. Figure 1 shows also that the self-trained readers tended to place more films into the central subcategories $(0 / 0,1 / 1,2 / 2$, and $3 / 3)$ of the four main categories than into the corresponding adjacent subcategories. Figure 2 shows the same trend in the experienced readers' classifications of films comprising a nominally uniform distribution to categories $\mathbf{0}$,

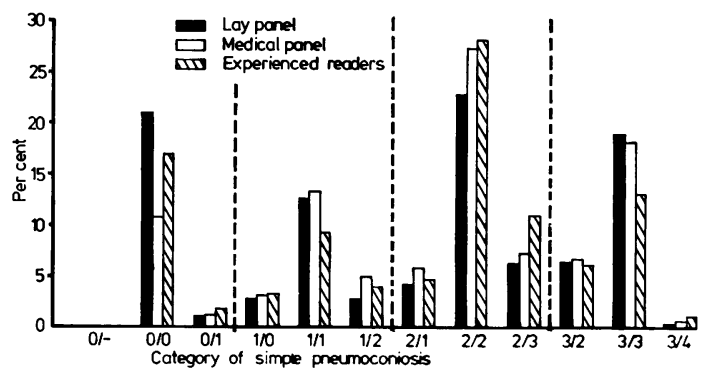

Fig 2 Lay, medical, and experienced readers' pooled distributions of a subset of 120 test radiographs to pneumoconiosis categories for small rounded opacities; experimental panels' third readings. 
Table 2 Percentage distributions of 220 radiographs by 24 readers to categories of profusion of small rounded opacities from the third readings by the experimental panels and earlier classifications by the experienced readers

\begin{tabular}{|c|c|c|c|c|c|}
\hline & \multirow{2}{*}{$\begin{array}{l}\text { No of } \\
\text { valid } \\
\text { classifications* }\end{array}$} & \multicolumn{4}{|c|}{ Category } \\
\hline & & 0 & 1 & 2 & 3 \\
\hline \multicolumn{6}{|l|}{ Medical panel } \\
\hline & $\begin{array}{l}216 \\
217 \\
145 \\
218 \\
220 \\
183 \\
218 \\
215\end{array}$ & $\begin{array}{r}26.4 \\
9 \cdot 7 \\
0.7 \\
21 \cdot 1 \\
17 \cdot 3 \\
0 \cdot 0 \\
7 \cdot 3 \\
7 \cdot 4\end{array}$ & $\begin{array}{l}21 \cdot 3 \\
39 \cdot 2 \\
31 \cdot 0 \\
14 \cdot 7 \\
45 \cdot 4 \\
23 \cdot 5 \\
17 \cdot 0 \\
20.9\end{array}$ & $\begin{array}{l}30 \cdot 1 \\
37 \cdot 8 \\
51 \cdot 7 \\
38 \cdot 1 \\
25 \cdot 9 \\
44 \cdot 8 \\
65 \cdot 6 \\
57 \cdot 2\end{array}$ & $\begin{array}{l}22 \cdot 2 \\
13 \cdot 4 \\
16 \cdot 6 \\
26 \cdot 1 \\
11 \cdot 4 \\
31 \cdot 7 \\
10 \cdot 1 \\
14 \cdot 4\end{array}$ \\
\hline All medical panel & 1632 & $11 \cdot 9$ & $26 \cdot 5$ & $43 \cdot 5$ & $18 \cdot 0$ \\
\hline \multicolumn{6}{|l|}{ Lay panel } \\
\hline & $\begin{array}{l}219 \\
217 \\
217 \\
217 \\
219 \\
216 \\
156 \\
217\end{array}$ & $\begin{array}{r}17 \cdot 8 \\
6 \cdot 4 \\
7 \cdot 4 \\
14 \cdot 7 \\
55 \cdot 2 \\
34 \cdot 3 \\
21 \cdot 2 \\
26 \cdot 3\end{array}$ & $\begin{array}{r}30 \cdot 6 \\
31 \cdot 3 \\
13 \cdot 4 \\
24.9 \\
8.7 \\
19 \cdot 4 \\
23 \cdot 7 \\
17 \cdot 5\end{array}$ & $\begin{array}{l}35 \cdot 2 \\
51 \cdot 2 \\
60 \cdot 4 \\
32 \cdot 3 \\
24 \cdot 2 \\
29 \cdot 6 \\
32 \cdot 7 \\
36 \cdot 4\end{array}$ & $\begin{array}{l}16.4 \\
11 \cdot 1 \\
18 \cdot 9 \\
28 \cdot 1 \\
11 \cdot 9 \\
16 \cdot 7 \\
22 \cdot 4 \\
19 \cdot 8\end{array}$ \\
\hline All lay panel & 1678 & $23 \cdot 0$ & $21 \cdot 1$ & $37 \cdot 9$ & $18 \cdot 0$ \\
\hline \multicolumn{6}{|l|}{ Experienced readers } \\
\hline & $\begin{array}{l}220 \\
220 \\
220 \\
220 \\
220 \\
220 \\
220 \\
220\end{array}$ & $\begin{array}{l}10 \cdot 0 \\
32 \cdot 3 \\
35 \cdot 9 \\
17 \cdot 3 \\
35 \cdot 4 \\
17 \cdot 3 \\
17 \cdot 7 \\
30 \cdot 0\end{array}$ & $\begin{array}{l}34 \cdot 5 \\
20 \cdot 4 \\
22 \cdot 7 \\
25 \cdot 4 \\
27 \cdot 3 \\
15 \cdot 9 \\
43 \cdot 2 \\
22 \cdot 3\end{array}$ & $\begin{array}{l}39 \cdot 5 \\
42 \cdot 7 \\
32 \cdot 7 \\
33 \cdot 2 \\
27 \cdot 3 \\
55 \cdot 9 \\
35 \cdot 9 \\
23 \cdot 2\end{array}$ & $\begin{array}{r}15 \cdot 9 \\
4 \cdot 5 \\
8 \cdot 6 \\
24 \cdot 1 \\
10 \cdot 0 \\
10 \cdot 9 \\
3 \cdot 2 \\
24 \cdot 5\end{array}$ \\
\hline All experienced readers & 1760 & $24 \cdot 5$ & $26 \cdot 5$ & $36 \cdot 3$ & $12 \cdot 7$ \\
\hline
\end{tabular}

* Reading sheets not completed precisely according to protocol were regarded as invalid and were disregarded in analyses of results.

$1,2,3, \mathrm{~A}$, and $\mathrm{B}$. This phenomenon has been observed also in other studies. ${ }^{6-10}$

Variability among readers in their allocations of radiographs to the four main categories of profusion is shown in table 2 using the subset of 220 radiographs that had been presented to all three groups. Maverick results occurred more often in the selftrained groups. Valid classifications from two of the medical panel were nearly all as category 1 or higher, and one lay reader considered more than half of 219 radiographs that she saw as category 0 . The latter reader's performance contributes largely to the higher range of results produced by the lay panel and to the closer similarity of their pooled distribution to that recorded by the experienced readers. In general, the distributions of classifications by the experienced readers are clustered closer together than those from the novice readers.

Even when two or more readers place identical numbers of films in particular categories this does not necessarily imply that they agree about the classification of individual radiographs. The extent of agreement between pairs of readers in their judgments about individual films, using the 12 categories of the complete classification, was measured by a consistency coefficient. This was defined as the number of films classified identically by any two readers, expressed as a percentage of the number assessed validly by both of them.

The medically qualified novice readers disagreed more among themselves than did the lay readers. The consistency coefficient from the third readings of the 300 test radiographs averaged $31 \%$ for the medical panel and $40 \%$ for the lay panel. Average consistency for 120 radiographs selected because they represented relatively high consensus between experienced readers was lower in the two selftrained panels (table 3). The reverse tendency was apparent for the other 100 films included on the basis of disagreements between the experienced readers concerning classifications to categories 0 and 1, and 1 and 2. For all 220 films consistency was 
Table 3 Mean between-reader consistency (\%) in classifications of small rounded opacities using the 12-point scale

\begin{tabular}{|c|c|c|c|}
\hline & Lay panel & $\begin{array}{l}\text { Medical } \\
\text { panel }\end{array}$ & $\begin{array}{l}\text { Experienced } \\
\text { readers }\end{array}$ \\
\hline $\begin{array}{l}120 \text { radiographs represent } \\
\text { agreements between }\end{array}$ & & & \\
\hline $\begin{array}{l}\text { experienced readers } \\
100 \text { radiographs represent } \\
\text { disagreements between }\end{array}$ & $g^{34 \cdot 5}$ & $28 \cdot 2$ & $42 \cdot 7$ \\
\hline $\begin{array}{l}\text { experienced readers } \\
\text { All (220) radiographs }\end{array}$ & $\begin{array}{l}26 \cdot 5 \\
30 \cdot 8\end{array}$ & $\begin{array}{l}25 \cdot 9 \\
27 \cdot 2\end{array}$ & $\begin{array}{l}15 \cdot 9 \\
30 \cdot 5\end{array}$ \\
\hline
\end{tabular}

(Figures quoted are averages of 28 comparisons between pairs of readers within groups.)

similar for all three groups.

Each of the 16 new readers placed some of the 60 films from men with no occupational dust exposure into categories $0 / 1$ or higher, at least on one occasion. At the third readings $16 \%$ of 875 valid classifications from all 16 readers were in category 1 or higher. Figure 3 summarises results from the repeated viewings in terms of the proportions classified as other than category $0 /-$ or $0 / 0$. On average, the lay readers were able to distinguish normal lung markings from radiographic evidence of dust lesions more easily than the doctors. The three radiographers performed better than their colleagues in this respect. One of them assessed all 60 films as category $0 / 0$ at

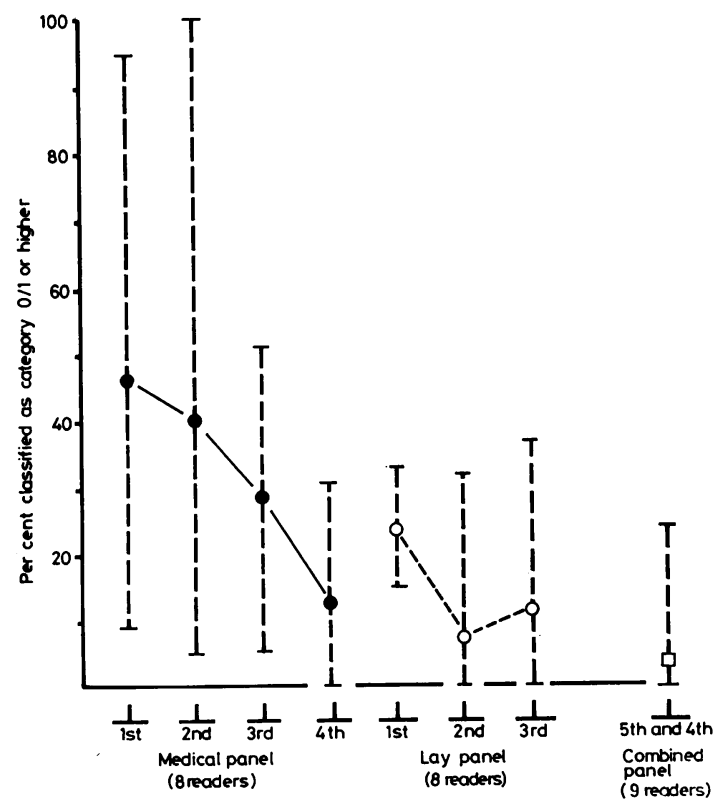

Fig 3 Mean results from repeated viewings of 60 radiographs of men with no occupational dust exposure. -- - - indicates ranges.
Table 4 Classifications of large opacities at the third readings

\begin{tabular}{lcc}
\hline & \multicolumn{2}{c}{ Experienced readers' judgments } \\
\cline { 2 - 3 } & $\begin{array}{l}\text { PMF } \\
(60 \text { films })\end{array}$ & $\begin{array}{l}\text { No PMF } \\
(240 \text { films })\end{array}$ \\
\hline $\begin{array}{l}\text { Medical panel's } \\
\text { classifications }\end{array}$ & & \\
Large opacities & 400 & 49 \\
No large opacities & 43 & 1818 \\
Total & 443 & 1867 \\
Lay panel's & & \\
classifications & & \\
Large opacities & 431 & 60 \\
No large opacities & 48 & 1849 \\
Total & 479 & 1909 \\
\hline
\end{tabular}

both second and third readings. The other two each placed one film into category 1 at the second reading, but judged two and seven films respectively as category 1 at the third readings. The medical panel spontaneously reduced the frequency of reading small rounded opacities in these radiographs over the three repeated viewings, but on average still decided that a quarter of them exceeded category $0 / 0$ at the third readings.

Sixty films had been included among the 300 because they illustrated large opacities. Ninety per cent of both experimental panels' classifications of these radiographs (at the third readings) included categorisation of large shadows (table 4). Conversely, classifications of 240 radiographs where no large opacities had been recorded by the experienced readers included $3 \%$ where members of the experimental panels noted large shadows.

Correlations between classifications of 792 other radiographs and coal miners' dust exposures were studied using scores corresponding to the natural numbers to represent the 12 categories of increasing profusion of small rounded opacities. ${ }^{7}$ The arithmetic mean of such scores from various readers' classifications of the same radiograph may be used to attribute an "average category" to the radiograph concerned. ${ }^{10}$ Complete results were available from the experienced readers for all 792 films, but there were missing data for some of the nine readers in the residual experimental panel. On average, the experienced readers classified nearly $80 \%$ of the 792 radiographs into category $0 / 0$. Only $29 \%$ (of 623 of these films) were so classified by the residual panel. The panel's tendency to classify far more films as showing small opacities is illustrated in figs 4 and 5, using four different indices of pneumoconiosis. In each case the average level of pneumoconiosis recorded by the residual panel is higher than that obtained by the experienced readers. But both groups of readers show clear correlations between 

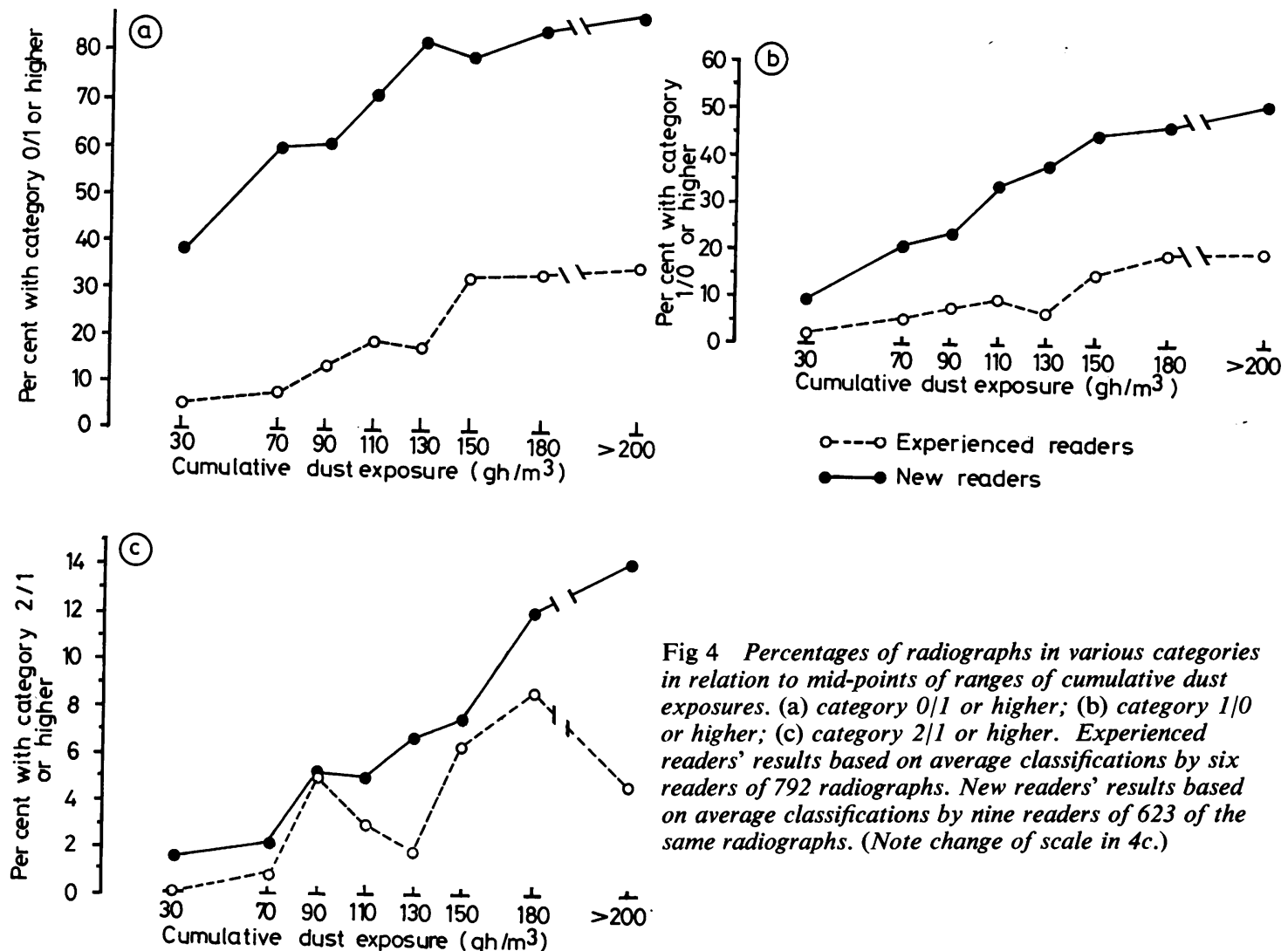

Fig 4 Percentages of radiographs in various categories in relation to mid-points of ranges of cumulative dust exposures. (a) category 0/1 or higher; (b) category $1 / 0$ or higher; (c) category 2/1 or higher. Experienced readers' results based on average classifications by six readers of 792 radiographs. New readers' results based on average classifications by nine readers of 623 of the same radiographs. (Note change of scale in $4 c$.)

cumulative exposures to dust and the four measures of radiological response. The correlations indicated are unlikely to be due to chance; the correlation coefficients between the miners' dust exposures and the mean pneumoconiosis scores (based on 790 and 621 degrees of freedom respectively) were 0.209 for the experienced readers and 0.265 for the residual

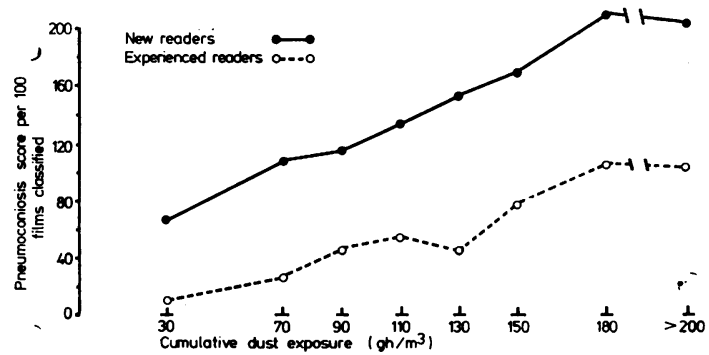

Fig 5 Mean pneumoconiosis scores in relation to midpoints of ranges of cumulative dust exposures. panel. Similar coefficients calculated from individual readers' pneumoconiosis scores were all positive, and all except one, from a novice reader, were statistically significant at the $2 \%$ level.

Between-reader variability was again higher among the nine panelists than among the six experienced readers. Pneumoconiosis scores, calculated from individual readers' results and averaged over all dustexposure groups, ranged from 25 to 290 among the panelists; the experienced readers' averages ranged from 38 to 84 . More detailed numerical results are available from the Institute of Occupational Medicine (report No TM/80/6). ${ }^{11}$

\section{Discussion}

Our readers were asked to classify films according to the complete ILO U/C classification. ${ }^{2}$ This includes provision for recording profusion of small irregular opacities and the presence of pleural abnormalities. Comments regarding other suspected disease may also be noted. Results reported here have been 
confined to classifications of small rounded opacities and of large shadows-that is, the most common radiographic signs of dust retention in the coal workers whose radiographs were studied.

Experienced physicians often disagree on how to describe the profusion of small opacities that they see on chest radiographs. ${ }^{10}$ This is why multiple readings, by more than just a single reader, are now usually regarded as mandatory in epidemiological studies using chest films. But replicating classifications of large numbers of radiographs is timeconsuming; it would be useful if people other than doctors specialising in occupational lung diseases could do this work. Our studies were designed to test whether it is possible to develop the necessary skills without simply reproducing the personal reading pattern of any one expert, since this may differ systematically from patterns adopted by other experts. The answer seems to be yes, at least so far as coal workers' pneumoconiosis is concerned. With practice, but without criticism or guidance as to what progress they were making, both our panels of novices produced classifications of small rounded opacities that generated, collectively, sensible distributions of radiographs to categories. In addition, the panels' ability to recognise large opacities compares reasonably with results from experienced readers in a large-scale epidemiological study. ${ }^{12}$

The descriptive text published by the ILO includes an important instruction requiring a reader to make an initial interpretative judgment as to whether "any of the changes seen in the pleura or the parenchyma are sufficiently characteristic of pneumoconiosis to be recorded." This text was deliberately and specifically relegated to a subsidiary role in the experimental protocol: the primary determinant in classifying a film was to be the similarity of its appearance to one or other of the standard radiographs issued by the ILO in 1971. The success of the experiments under these conditions therefore shows that the ILO scale of profusion, as elaborated along the lines first recommended by Liddell, ${ }^{6}$ can be applied as a purely descriptive tool; neither clinical experience nor competence in diagnosis of chest diseases is a necessary condition. An important corollary is that the results must not be interpreted as evidence that lay people, or doctors with no special training in chest radiology, are able to diagnose occupational lung diseases. (All the radiographs used in the experiments described had been examined previously for clinical purposes by appropriately qualified doctors.) Nevertheless, for epidemiological purposes, it is precisely descriptions of radiographs (rather than radiological diagnoses) that are of interest. For if these descriptions are made systematically, according to defined rules, then they may be used validly to quantify radiographic appearances in groups of people. This distinction between clinical assessments and epidemiological work with chest radiographs is reflected in the American Thoracic Society's proproposals for epidemiological studies of nonoccupational lung diseases. ${ }^{13}$ The authors of that report note that important epidemiological data may be lost if film readers are asked to classify only those radiographs where they judge the appearances to be due to pneumoconiosis. Our view is that the same principle applies when the aim is to classify radiographs for pneumoconiosis; but this remains a controversial question. ${ }^{14}$

The variability between new readers in their distributions of films to categories was higher than among the experienced readers. This was due mainly to three of the sixteen novices. But the average consistency between pairs of readers in their classifications of individual films was similar in all three groups.

Nevertheless, the panels' classifications of the 60 radiographs from men with no occupational exposure to dust shows a potential difficulty in accepting a purely descriptive categorisation of appearances of chest films. Normal vascular shadows, and small opacities possibly associated with aging or tobacco consumption, may be classified conscientiously, according to their profusion. The results might be interpreted wrongly as indicating pneumoconiosis. This could be a seriously disturbing factor is epidemiological studies. Medically qualified readers experienced in diagnosis of lung diseases are less likely to make such errors. In this connection it is interesting that the radiographers only rarely classified these 60 radiographs higher than category $0 / 0$. The medical panel performed less satisfactorily, particularly at their initial readings. But they showed an apparently spontaneous "learning effect," in that on repeated viewing they steadily decreased the proportion of these 60 films that they classified into category $0 / 1$ or higher. This decrease occurred in the absence of any "feedback" of information that might have influenced them. It appears therefore that practice was important. The readers had their attention drawn to the problem only after the initial experiments (the third and fourth readings by the medical and lay panels respectively). They were then shown further examples of radiographs (not included among the 300) of people known definitely to have had no occupational dust exposure. This is not the same as instructing learners on how to classify films from people who have been exposed to dust. But it was probably useful, because most of the nine members of the residual panel performed acceptably in this respect at their 
subsequent viewing of the test radiographs. A helpful recent development is the inclusion of a second film illustrating category 0/0 in the ILO's new (1980) set of standard radiographs.

In this first account of the work of these panels no data have been presented on within-reader consistency - that is, on consistency of classifications of the same radiographs by any one reader on repeated viewings. This is because the replicate classifications described here were generated as part of the selftraining protocol; the results would therefore be uninterpretable as indices of long-term stability in reading habits. Intra-observer repeatability, however, is an important measure for judging the reliability of epidemiological data. It is being monitored as part of the continuing studies involving some of these readers.

Some of the difference between the levels of pneumoconiosis recorded by the new and the experienced readers may be attributable to the fact that the novices worked strictly to standard films issued by the ILO in 1971. The experienced readers' classifications were all based on earlier sets of standards, and many experts have remarked on faulty photocopying technique in some of the 1971 sets distributed.

In the last analysis the success or failure of these experiments may be judged by readers' ability to classify radiographs in a way that produces convincing and informative correlations with an objective criterion. Cumulative exposure to airborne dust is such a criterion. We have shown that readers trained in the way described may generate valid data for epidemiological studies. Clearly, it will be desirable, perhaps even essential, that the performances of such readers should be monitored periodically by comparisons with results from medically qualified experts, but such checks are necessary also in other radiological studies if results from one research group are to be compared validly with those from another.

The experiments with the lay panel were supported by a grant from the Medical Research Council. Other costs were met by the National Coal Board. The classifications used to select the test radiographs were provided by past and present members of the National Coal Board Medical Service's team of experts. We are very grateful to Drs J G Bennett, J A Dick, D J Jones, D C Morgan, S Rae, D J Thomas, D A Walker, and J S Washington. Drs J G Bennett, J Burns, J A Dick, D A Scarisbrick, D J Thomas, and
J S Washington made the classifications used for comparisons of correlations with dust exposure.

We pay tribute to the patient and careful work of our panels: Drs J G Van Bavel, I S Fenton, M R Fettes, S J Hadfield, A H McDonald, M Price, D R Redman, and L Warwick; and Mrs H G Corsie, Mrs K Duncan, Dr F J Elliot, Mrs E Henderson, Mrs A F Primrose, Mrs S E Quinn, Mrs C Scott, and Mrs V G White.

\section{References}

${ }^{1}$ Peters WL, Reger RB, Morgan WKC. The radiographic categorisation of coalworkers' pneumoconiosis by lay readers. Environ Res 1973;6:60-7.

${ }^{2}$ International Labour Office. ILO U/C International classification of radiographs of pneumoconioses 1971. Geneva: ILO, 1972. (Occupational Safety and Health Series No 22 (rev).)

${ }^{3}$ Jacobsen M, Rae S, Walton WH, Rogan JM. The relation between pneunioconiosis and dust exposure in British coal mines. In: Walton $\mathrm{WH}$, ed. Inhaled particles $I I I$. Old Woking, Surrey: Unwin, 1971;2:903-19.

${ }^{4}$ Fay JWJ, Ashford JR. The study of observer variation in the radiological classification of pneumoconiosis. $\mathrm{Br} J$ Ind Med 1960;17:279-92.

5 Muir DCF, Burns J, Jacobsen M, Walton WH. Pneumoconiosis and chronic bronchitis. $\mathrm{Br}$ Med $J$ 1977; ii : 424-7.

${ }^{6}$ Liddell FDK. An experiment in film reading. Br $\mathrm{J}$ Ind $\mathrm{Med}$ $1963 ; 20: 300-12$.

7 Liddell FDK, Lindars DC. An elaboration of the ILO classification of simple pneumoconiosis. $\mathrm{Br} J$ Ind $\mathrm{Med}$ 1969;26:89-100.

${ }^{8}$ Rossiter CE. Relation between content and composition of coalworkers' lungs and radiological appearances. $\mathrm{Br} J$ Ind Med 1972;29:31-44.

9 Morgan WKC, Petersen MR, Reger RB. The "middling" tendency: a possible source of bias in the interpretation of chest films for pneumoconiosis. Arch Environ Health $1974 ; 29: 334-7$

10 Jacobsen M. Quantifying radiological changes in simple pneumoconiosis. Applied Statistics 1975;24:229-49.

11 Copland L, Burns J, Jacobsen M. Classification of chest radiographs for epidemiological purposes by persons not experienced in the radiology of pneumoconiosis. Edinburgh: Institute of Occupational Medicine, 1980. (Report No TM/80/6.)

12 McLintock JS, Rae S, Jacobsen M. The attack rate of progressive massive fibrosis in British coalminers. In: Walton WH, ed. Inhaled particles III. Old Woking, Surrey: Unwin, $1971 ; 2: 933-52$.

13 American Thoracic Society. Use of chest radiography in epidemiological studies of non-occupational lung diseases. Am Rev Respir Dis 1978;118:No 6, pt 2:89-111.

${ }^{14}$ Gilson JC, Jacobsen M. Recommendations for future research. Non-pneumoconiotic shadows. In: International Labour Office Guidelines for the use of the ILO International Classification of Radiographs of Pneumoconioses. Geneva: ILO, 1980:33-4. (Revised edition 1980.) 